\title{
Recent Trends in the Surgical Treatment of Secondary Hyperparathyroidism
}

\author{
Ho-Ryun Won (D) B Bon Seok Koo id \\ Department of Otolaryngology-Head and Neck Surgery, Chungnam National University College of Medicine, Daejeon, Korea
}

Secondary hyperparathyroidism (SHPT) is a common complication in chronic kidney disease (CKD) patients undergoing dialysis, as it is reported to occur in approximately $86 \%$ of cases [1]. The main pathophysiological mechanism of SHPT in CKD patients is hyperphosphatemia, which occurs because the reduction of the glomerular filtration rate due to CKD decreases the phosphorus clearance rate. In hyperphosphatemia, 1,25-dihydroxyvitamin D levels are reduced by an increase in fibroblast growth factor 23 levels, which eventually leads to hypocalcemia. This hypocalcemic state consequently stimulates the parathyroid gland to sustain the secretion of parathyroid hormone (PTH). If this process continues due to a deterioration of renal function, a vicious cycle of hyperphosphatemia inducing increased PTH levels continues, as PTH has the effect of reducing phosphorus resorption [2].

If SHPT persists uncontrolled, osteodystrophy increases, thereby resulting in a higher risk of fracture. In addition, the increased deposition of calcium in blood vessels and tissues results in a higher risk of cardiovascular disease. PTH exerts a direct adverse effect on the bone marrow, resulting in resistant anemia with hyporesponsiveness to erythropoiesis. Despite many advances in dialysis treatment, there has been no significant change in mortality in CKD patients, and complications from SHPT are the leading cause of death. Therefore, proper control and treatment of SHPT in CKD patients are of vital importance [2].

The first-line treatment for SHPT is pharmacological treatment involving phosphorus binders, calcimimetics, calcitriol, and vitamin D analogs, including vitamin D mimetics. If there is no response or severe side effects to pharmacological treatment, surgical treatment should be considered. According to the 2017 Kidney Disease: Improving Global Outcomes guidelines, surgical treatment is recommended for patients at all stages, including the early stages of CKD, when there is no response to pharmacological treatment [3]. The surgical treatment of SHPT has gradually become more common in recent years, and the Japanese Society for Dialysis Therapy reported that parathyroidectomy was performed in $10 \%$ of patients undergoing dialysis for 10 years and $30 \%$ of patients undergoing dialysis for 20 years [4].

Surgical treatment methods for patients with SHPT have been studied for a long time, generating an extensive literature. The currently available surgical methods are subtotal parathyroidectomy (SPTX), total parathyroidectomy (TPTX), and TPTX with autotransplantation (TPTX+AT). Each surgical method has advantages and disadvantages, and controversy remains regarding which method is preferable. SPTX was first reported in 1960 and is a technique that removes three and a half glands, leaving about 40-80 mg of normal parathyroid gland tissue. SPTX has been confirmed to be effective in treating SHPT in a recently published paper by Kim et al. [5], and tends to be more widely preferred in the United States. In TPTX+AT, the entire parathyroid gland is removed, and then some parathyroid tissue is collected and transplanted into the sternocleidomastoid muscle, trapezius muscle, or brachioradialis muscle. This technique is relatively common in Japan. TPTX is selectively performed in patients at high risk for reoperation $[2,4]$.

The most restrictive point of SPTX is the possibility of recurrence of SPHT caused by the remaining parathyroid tissue, and TPTX has been proposed to overcome this disadvantage. However, there is a possibility of permanent hypoparathyroidism and consequent hypocalcemia, which motivated the proposal of TPTX+AT. According to a meta-analysis published in 2017 by Chen et al. [6], there were no statistically significant differences in recurrence, persistence, or the reoperation rate between SPTX and TPTX+AT. In 2016, a multicenter, prospective randomized controlled trial was performed to compare TPTX and TPTX+AT in patients with SPHT by Schlosser et al. [7], and it was reported that TPTX reduced PTH levels more effectively than TPTX+AT. However, it was suggested that more research is needed on the recurrence rate. Similarly, Liu et al. [8], in a meta-analysis comparing TPTX and TPTX+AT, reported that TPTX is effective in reducing $\mathrm{PTH}$, but more extensive studies are needed to evaluate the recurrence rate.

Copyright (C) 2020 by Korean Society of Otorhinolaryngology-Head and Neck Surgery.

This is an open-access article distributed under the terms of the Creative Commons Attribution Non-Commercial License (https://creativecommons.org/licenses/by-nc/4.0)

which permits unrestricted non-commercial use, distribution, and reproduction in any medium, provided the original work is properly cited. 
In conclusion, the optimal surgical treatment method for the treatment of SHPT remains controversial. Therefore, when determining the surgical method, the clinician's judgment and patient-related factors are of vital importance. SPTX or TPTX+AT may be considered as a priority for patients with CKD who are expected to undergo renal transplantation in the future, and TPTX or TPTX+AT may be preferred if the patient has a high risk of reoperation of the neck. TPTX may be useful in patients with no renal transplantation potential and a long life expectancy. Most importantly, in order to reduce the mortality rate of CKD patients, the most important factor is not which surgical technique is selected, but the optimal timing of surgical treatment through a multi-disciplinary approach.

\section{CONFLICT OF INTEREST}

No potential conflict of interest relevant to this article was reported.

\section{ORCID}

Ho-Ryun Won https://orcid.org/0000-0002-5135-2474

Bon Seok Koo https://orcid.org/0000-0002-5928-0006

\section{REFERENCES}

1. Yang G, Zhang B, Zha XM, Wang NN, Xing CY.Total parathyroidectomy with autotransplantation for a rare disease derived from ure- mic secondary hyperparathyroidism, the uremic leontiasis ossea. Osteoporos Int. 2014 Mar;25(3):1115-21.

2. Lau WL, Obi Y, Kalantar-Zadeh K. Parathyroidectomy in the management of secondary hyperparathyroidism. Clin J Am Soc Nephrol. 2018 Jun;13(6):952-61.

3. Ketteler M, Block GA, Evenepoel P, Fukagawa M, Herzog CA, McCann L, et al. Executive summary of the 2017 KDIGO Chronic Kidney Disease-Mineral and Bone Disorder (CKD-MBD) Guideline Update: what's changed and why it matters. Kidney Int. 2017 Jul;92(1): 26-36.

4. Tominaga Y, Uchida K, HabaT, Katayama A, SatoT, HibiY, et al. More than 1,000 cases of total parathyroidectomy with forearm autograft for renal hyperparathyroidism. Am J Kidney Dis. 2001 Oct;38(4 Suppl 1):S168-71.

5. Kim MS, Kim GH, Lee CH, Park JS, Lee JY,Tae K. Surgical outcomes of subtotal parathyroidectomy for renal hyperparathyroidism. Clin Exp Otorhinolaryngol. 2020;13(2):173-8.

6. Chen J, Jia X, Kong X, Wang Z, Cui M, Xu D.Total parathyroidectomy with autotransplantation versus subtotal parathyroidectomy for renal hyperparathyroidism: a systematic review and meta-analysis. Nephrology (Carlton). 2017 May;22(5):388-96.

7. Schlosser K, Bartsch DK, Diener MK, Seiler CM, BrucknerT, Nies C, et al. Total parathyroidectomy with routine thymectomy and autotransplantation versus total parathyroidectomy alone for secondary hyperparathyroidism: results of a nonconfirmatory multicenter prospective randomized controlled pilot trial. Ann Surg. 2016 Nov; 264(5):745-53.

8. Liu ME, Qiu NC, Zha SL, Du ZP, Wang YF, Wang Q, et al.To assess the effects of parathyroidectomy (TPTX versus TPTX+AT) for Secondary hyperparathyroidism in chronic renal failure: a systematic review and meta-analysis. Int J Surg. 2017 Aug;44:353-62. 